\title{
Asymptomatic Anisakis and erosive lesions in the colon
}

\section{Angelo Zulloa, Giuseppina Balsamob, Daniela Baldini' ${ }^{b}$, Vincenzo De Francescoc, Raffaele Mantad}

Nuovo Regina Margherita Hospital, Rome; Riuniti Hospital, Foggia; Sant' Agostino-Estense Hospital, Baggiovara-Modena, Italy

A 50-year-old, asymptomatic man underwent scheduled screening colonoscopy. Because of his first-degree family history for colorectal cancer, he had previously undergone a colonoscopy 5 years before, but no mucosal alterations were found. A total colonoscopy was performed without difficulty under conscious sedation with midazolam $5 \mathrm{mg}$ i.v., although mild pain occurred when the colonoscope passed through the sigmoid tract. Withdrawal of the endoscope also elicited pain in the sigmoid, where some mucosal folds appeared to be moderately enlarged. When the tract was more closely observed, a mobile nematode was discovered among the folds, showing extremely strong adherence to the mucosa (Fig. 1A). The worm was grasped and successfully removed with a forceps. In the adjacent mucosa, we observed some slight elevated nodules with apical depression (Fig. 1B). Histological examination of these lesions showed a marked infiltration of eosinophils in the colonic mucosa (Fig. 1C). The removed nematode was characterized as $18-\mathrm{mm}$ long Anisakis simplex (Fig. 1D). The patient disclosed that he had eaten uncooked anchovies 5 days before. No symptoms occurred at 3-month follow up.

Endoscopic diagnosis of Anisakis in the stomach and in the colon has been reported in symptomatic patients [1-3].

a'Gastroenterology Unit (Angelo Zullo); 'Pathology Unit (Giuseppina Balsamo, Daniela Baldini), 'Nuovo Regina Margherita' Hospital, Rome; 'Gastroenterology Unit, 'Riuniti' Hospital, Foggia (Vincenzo De Francesco); 'Gastroenterology Unit, 'Sant'Agostino-Estense' Hospital, Baggiovara-Modena (Raffaele Manta), Italy

Conflict of Interest: None

Correspondence to: Angelo Zullo,

Ospedale Nuovo Regina Margherita, Gastroenterologia ed Endoscopia Digestiva, Via Emilio Morosini, 3000153 Rome, Italy

E-mail: angelozullo66@yahoo.it

Received 24 October 2017; accepted 30 October 2017; published online 27 November 2017

DOI: https://doi.org/10.20524/aog.2017.0216

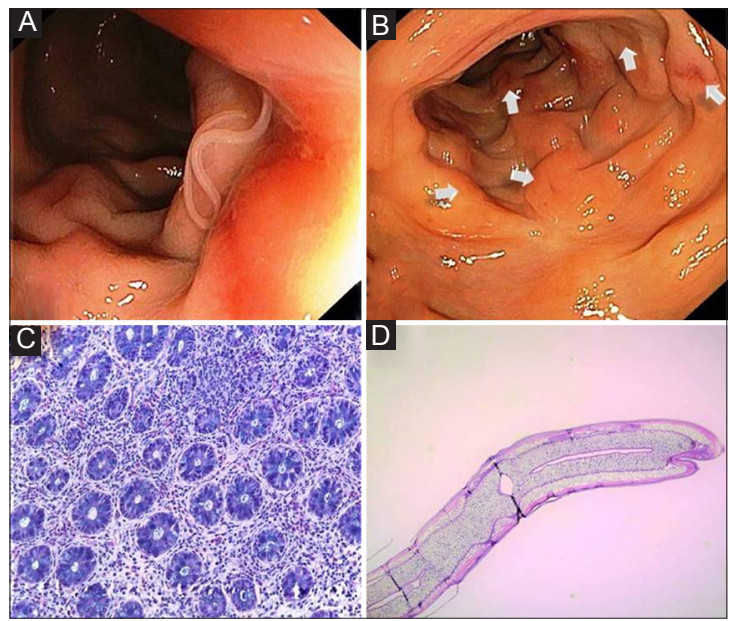

Figure 1 (A) A mobile nematode detected on the sigmoid mucosa. (B) Erosive lesions on the adjacent mucosa. (C) Histological examination of erosions showing infiltration of eosinophils. (D) Microscopic observation showing Anisakis simplex

However, to our knowledge, only 4 cases of anisakiasis detection in the colon in asymptomatic subjects have previously been described [3]. We would suggest that the adjacent nodular mucosal alterations may represent the result of unsuccessful attempts of the same worm-or other similar ones, probably eliminated during bowel preparation-to fix on the mucosa.

\section{References}

1. Hamada K, Uedo N, Tomita Y, Iishi H. A bleeding gastric ulcer caused by anisakiasis. Ann Gastroenterol 2016;29:378.

2. Zullo A, Hassan C, Scaccianoce G, et al. Gastric anisakiasis: do not forget the clinical history! J Gastrointest Liver Dis 2010;19:359.

3. Taniguchi G, Nagahara A, Matsumoto K, et al. Asymptomatic anisakiasis of the colon incidentally found by colonoscopy. Clin J Gastroenterol 2011;4:371-373. 